\title{
Upregulation of FoxP4 in HCC promotes migration and invasion through regulation of EMT
}

\author{
GANG ZHANG and GUANGYE ZHANG
}

Department of Liver Diseases, Rizhao Hospital of Traditional Chinese Medicine, Rizhao, Shandong 276800, P.R. China

Received April 8, 2018; Accepted November 16, 2018

DOI: $10.3892 / \mathrm{ol} .2019 .10049$

\begin{abstract}
Previous studies have indicated that FoxP1, FoxP2 and FoxP3 play important roles in hepatocellular carcinoma (HCC). However, the effect of FoxP4 in HCC requires further elucidation. The aim of the present study was to explore the roles of FoxP4 in HCC and further decipher the detailed mechanism. In present study, it was found that FoxP4, which is overexpressed in HCC tissues and cell lines, facilitated EMT in HCC cell lines through regulation of Slug. First, increased expression of FoxP4 was identified in 110 pairs of human HCC tumor and their adjacent normal tissues. In addition, the association between FoxP4 expression and clinicopathological features of HCC patients indicated that FoxP4 played vital roles in HCC development. Subsequently, gain- and loss-of-function experiments indicated that FoxP4 promoted cellular proliferation, migration as well invasion. In addition, EMT, a key mechanism during cancer metastasis, was regulated by FoxP4. Furthermore, ChIP and qChIP as well as luciferase reporter assays indicated that Slug, an EMT-associated transcription factor, was transcriptionally regulated by FoxP4. In conclusion, FoxP4 functioned as a tumor promoter in HCC cells by transcriptionally regulating Slug, and the present study highlighted the potential effects of FoxP4 on the prognosis and treatment of HCC.
\end{abstract}

\section{Introduction}

Hepatocellular carcinoma (HCC) is the sixth most prevalent malignant tumor worldwide, with rising incidence in recent decades $(1,2)$. The development of surgical techniques has improved the prognosis of patients with HCC. However, due to high mortality (3), the overall survival rate of patients with HCC is still poor $(2,4)$. Notably, research has revealed that a variety of molecules play key roles in the initiation and

Correspondence to: Dr Guangye Zhang, Department of Liver Diseases, Rizhao Hospital of Traditional Chinese Medicine, 35 Wanghai Road, Rizhao, Shandong 276800, P.R. China E-mail: ZGY10102010@163.com

Key words: FoxP4, proliferation, EMT, Slug, hepatocellular carcinoma progression of HCC (5). Thus, finding reliable biomarkers to diagnose $\mathrm{HCC}$ and developing novel strategies are required for the effective treatment of patients with HCC.

Increasingly, evidence has indicated that different transcription factor (TF) classes play critical roles for tumor development (6). For example, the member of forkhead box $\mathrm{P}$ (FoxP) family, FoxP1, FoxP2 and FoxP3, have been revealed to play an important function in HCC (7-12). The FoxP subfamily which consists of four members (FoxP1-4) belongs to the Fox superfamily, it has a highly conserved 'winged-helix' or 'fork-head' $(13,14)$. Fox proteins are involved in cell cycle progression, apoptosis, proliferation as well as in senescence and metabolism (15). However, the function of FoxP4, the other member of the FoxP family, has yet to be revealed.

Epithelial-mesenchymal transition (EMT) is a complex process which is closely associated with the metastasis of cancer cells (16). The main characteristic of EMT is the transition of cell phenotype, epithelial cells change their phenotype from epithelial to mesenchymal (16). Previous studies have identified that several transcription factors are involved in EMT, including Slug, Snail and Twistl $(17,18)$. A recent study demonstrated that the member of the Fox family plays a crucial role in EMT (19).

The aim of the present study was to reveal the role of FoxP4 in HCC. Its functions in HCCLM3 cells were investigated with colony formation, CCK-8, wound healing and Transwell invasion assays. Moreover, ChIP and qChIP as well as a luciferase reporter assay demonstrated that Slug was a downstream target of FoxP4. In conclusion, our study revealed that FoxP4 plays a key role in HCC.

\section{Materials and methods}

Cell culture. Human HL-7702 normal liver cell line and human HCCLM3 cells were purchased from the Shanghai Institute of Biochemistry and Cell Biology, Shanghai Institutes for Biological Sciences, Chinese Academy of Sciences (Shanghai, China). Cell lines were cultured in DMEM (HyClone; GE Healthcare Life Sciences, Logan, UT, USA) supplemented with $1 \%$ penicillin and $1 \%$ streptomycin as well as $10 \%$ fetal bovine serum (FBS; HyClone; GE Healthcare Life Sciences, and cells were maintained at $37^{\circ} \mathrm{C}$ in a $5 \% \mathrm{CO}_{2}$ atmosphere.

Patients and tissue samples. Human HCC and their adjacent normal liver tissues (110 pairs) were collected from HCC 
patients undergoing liver resections at the Department of Liver Diseases, Rizhao Hospital of Traditional Chinese Medicine from April 2005 to December 2017. All tissues samples were collected from patients who had not received any antitumor therapy and tissue samples were stored in liquid nitrogen before use. The study was approved by the Ethics Committee of Rizhao Hospital of Traditional Chinese Medicine and each patient was well informed and signed informed consent forms.

Cell transfection. Vector (pcDNA3.1) and pcDNA3.1-FoxP4 were purchased from Vigene Biosciences (Shandong, China). Scramble siRNA (SCR) and FoxP4 siRNA (siFoxP4) were designed and synthesized by GenePharma (Shanghai, China). For cell transfection, the cells were transfected with $2.5 \mu \mathrm{g}$ plasmid or $50 \mathrm{nM}$ siRNA using Lipofectamine 2000 (Invitrogen; Thermo Fisher Scientific, Inc., Waltham, MA, USA) according to the manufacturer's protocol. After transfection for $48 \mathrm{~h}$, the cells were collected and used for the subsequent experiments.

Real-Time quantitative polymerase chain reaction (qRT-qPCR) analysis. Total RNA was extracted from tissue samples and cells using TRIzol reagent (Invitrogen; Thermo Fisher Scientific, Inc.) according to manufacturer's instructions. RNA $(2 \mu \mathrm{g})$ was reverse-transcribed into cDNA using the PrimeScript ${ }^{\mathrm{TM}}$ RT Reagent kit. qPCR was performed using SYBR Green (Roche Diagnostics, Mannheim, Germany). The thermocycling conditions were as follows: $95^{\circ} \mathrm{C}$ for $10 \mathrm{~min}$, followed by 35 cycles at $95^{\circ} \mathrm{C}$ for $60 \mathrm{sec}$ (denaturation), $56^{\circ} \mathrm{C}$ for $60 \mathrm{sec}$ (annealing), and $72^{\circ} \mathrm{C}$ for $2 \mathrm{~min}$ (extension), followed by $72^{\circ} \mathrm{C}$ for $6 \mathrm{~min}$. GAPDH served as an internal control. Relative gene expression was quantified using $2^{-\Delta \Delta \mathrm{Cq}}$ method (20).

Western blot analysis. Approximately $2 \times 10^{6}$ HCCLM3 cells were collected by centrifugation at $800 \times \mathrm{g}$ for $5 \mathrm{~min}$ at $4^{\circ} \mathrm{C}$, and lysed in RIPA buffer (10\% NP-40, $10 \%$ sodium deoxycholate, $100 \mathrm{mM} \mathrm{NaCl}, 20 \mathrm{mM}$ Tris- $\mathrm{HCl} \mathrm{pH} 7.4$ and $100 \mathrm{mM}$ EDTA) at $4^{\circ} \mathrm{C}$ for $30 \mathrm{~min}$. Samples were subsequently centrifuged at $13,000 \times \mathrm{g}$ for $15 \mathrm{~min}$ at $4^{\circ} \mathrm{C}$ and the supernatants were collected. The concentration of the proteins was assessed using a BCA protein assay (Pierce; Thermo Fisher Scientific, Inc.) according to manufacturer's protocol. Equal amounts of samples $(40 \mu \mathrm{g})$ were separated by $10 \%$ SDS-PAGE and transferred onto PVDF membranes. After blocking with 5\% non-fat milk for $1 \mathrm{~h}$ at room temperature, the membranes were incubated with the indicated antibodies overnight at $4^{\circ} \mathrm{C}$. Following washing with TBST solution three times, the membranes were incubated with HRP-conjugated secondary antibodies for $1 \mathrm{~h}$ at room temperature. The blots were visualized using enhanced chemiluminescence (Pierce; Thermo Fisher Scientific, Inc.). The antibodies used were as follows: rabbit anti-E-cadherin $(1: 1,000)$, rabbit anti-N-cadherin $(1: 1,000)$ and mouse anti- $\beta$-actin $(1: 1,000)$ antibodies (all from Abcam; Cambridge, MA, USA); rabbit/mouse secondary (1:5,000) antibodies (Proteintech; Wuhan Sanying Biotechnology, Wuhan, China).

CCK-8 assay. A CCK-8 assay was performed to establish the effect of FoxP4 on cellular proliferation. Briefly, $\sim 2 \times 10^{3}$ transfected cells were placed into 96-well plates with $200 \mu \mathrm{l}$ DMEM supplemented with $10 \%$ FBS, and $20 \mu \mathrm{l} \mathrm{CCK}-8$ solution (Beyotime Institute of Biotechnology, Shanghai, China) was added to each well after culturing for $0,24,48$ and $72 \mathrm{~h}$. Following incubation for another $30 \mathrm{~min}$ at $37^{\circ} \mathrm{C}$, the absorbance value was assessed at $450 \mathrm{~nm}$ using a microplate reader (Bio-Tek Instruments, Winooski, VT, USA). Each independent experiment was replicated at least three times.

Colony formation assay. Briefly, $\sim 5 \times 10^{3}$ transfected cells were cultured in 6 -well plates for 12 days. The medium was replaced every three days. After 12 days, the colonies were fixed with methanol at room temperature for $10 \mathrm{~min}$, and then stained with $0.1 \%$ crystal violet (Sigma-Aldrich; Merck $\mathrm{KGaA}$, Darmstadt, Germany) at room temperature for $15 \mathrm{~min}$. The number of colonies was counted under a light microscope. Each independent experiment was replicated at least three times.

Wound healing assay. A wound healing assay was performed to determine the effect of FoxP4 on the migratory capabilities of HCC cells. In brief, $4 \times 10^{5}$ transfected HCCLM3 cells were plated in 12-well plates and cultured in DMEM supplemented with $1 \%$ penicillin and $1 \%$ streptomycin as well as $10 \% \mathrm{FBS}$ at $37^{\circ} \mathrm{C}$ with $5 \% \mathrm{CO}_{2}$ until $90-100 \%$ confluence. A $20-\mu$ l pipette tip was utilized to scratch a line wound. The detached cells were removed using ice-cold PBS. Subsequently, the cells were cultured in serum-free DMEM at $37^{\circ} \mathrm{C}$ with $5 \% \mathrm{CO}_{2}$. The scratch wounds were observed at 0 and $48 \mathrm{~h}$ under a light microscope and photomicrographs were captured. The migration of cells was analyzed using ImageJ 1.48 software (National Institutes of Health, Bethesda, MD, USA). The wound healing rate was calculated according to the following formula: Wound healing rate $=[($ scratch width at $0 \mathrm{~h})$-scratch width at $48 \mathrm{~h}] /($ scratch width at $0 \mathrm{~h})]$ x $100 \%$. Each independent experiment was repeated at least three times.

Transwell invasion analysis. Transwell invasion analysis was used to determine cell migration and invasion capacities. Briefly, Transwell chambers with $8-\mu \mathrm{m}$ pore sizes (Corning, Cambridge, MA, USA) were coated with $80 \mu 1$ 1:16-diluted Matrigel-coated (BD Biosciences, Franklin Lakes, NJ, USA) and incubated at $37^{\circ} \mathrm{C}$ for $1 \mathrm{~h}$. After transfection for $24 \mathrm{~h}$, approximately $2.5 \times 10^{4}$ cells were placed into the upper chamber with $300 \mu \mathrm{l}$ serum-free DMEM medium, while $500 \mu 1$ DMEM supplemented with $10 \%$ FBS was added to the lower chamber. Following incubation for $48 \mathrm{~h}$, the cells in the upper chamber were removed by a cotton swab and the cells in the lower chamber were then stained with $0.1 \%$ crystal violet (Sigma-Aldrich; Merck KGaA) for 10 min at room temperature. Finally, the invaded cells were counted and photographed under a light microscope. Each independent experiment was replicated at least three times.

Dual luciferase reporter assay. For the dual luciferase reporter assay, the promoter region $(-2,000$ to +200$)$ of Slug was cloned into pGL3-basic plasmid, and 293T cells were placed in 12-well plates and co-transfected with pGL3-Slug, Renilla, FoxP4, FoxP4 siRNA or the corresponding negative control. After transcription for $24 \mathrm{~h}$, the cells were collected. The luciferase activity was assessed using a dual luciferase reporter assay system (Promega Corporation, Madison, WI, 

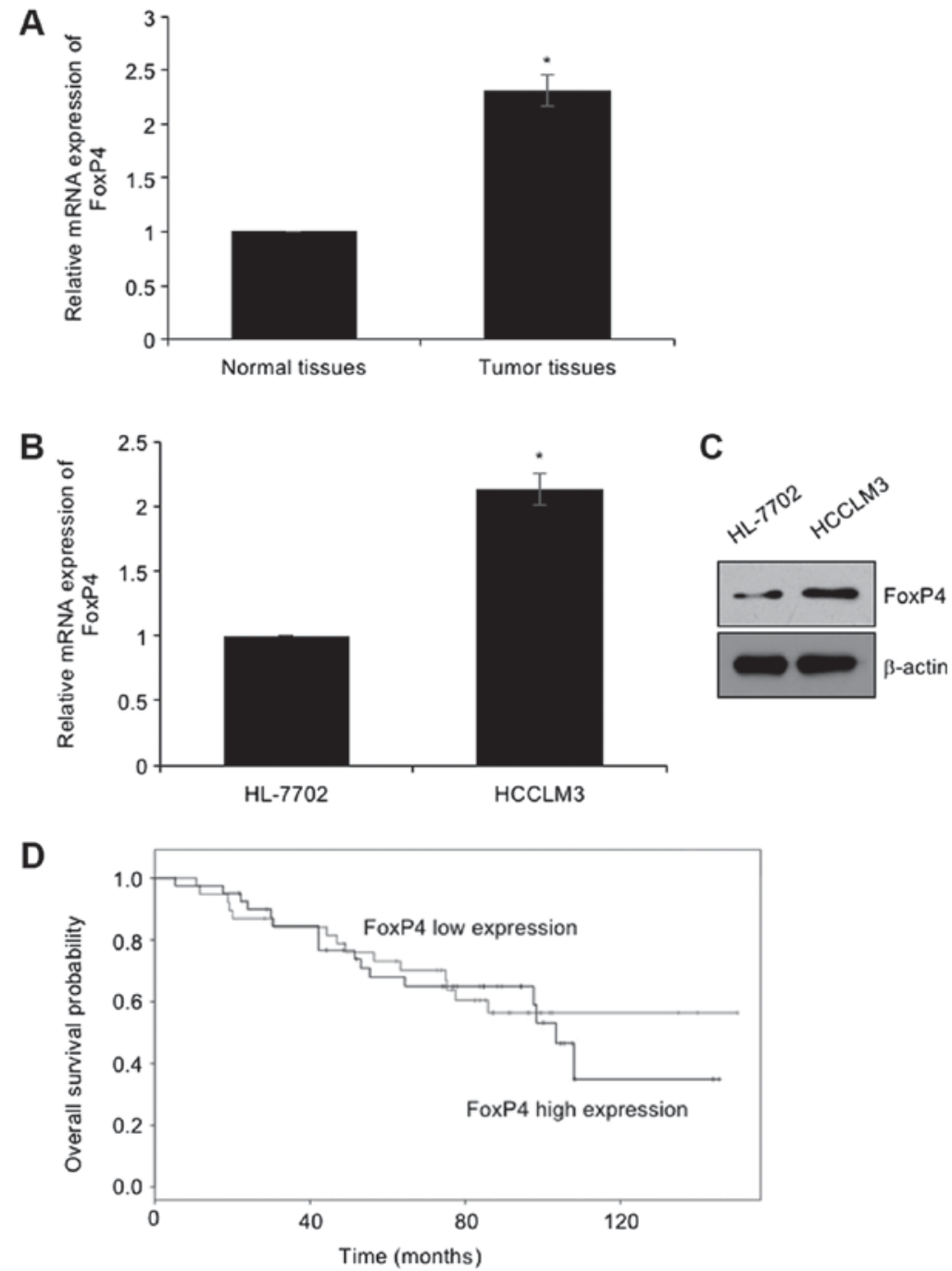

Figure 1. Expression of FoxP4 is elevated in HCC tissues and cell lines. (A) RT-qPCR was used to determine the expression of FoxP4 in the HCC tissues. (vs. Adjacent normal tissues, ${ }^{*} \mathrm{P}<0.05$ ). (B and C) RT-qPCR and western blotting assays were used to assess the mRNA and protein levels of FoxP4 in the HCCLM3 cell line and in the human HL-7702 normal hepatocyte line. (vs. HL-7702, ${ }^{*} \mathrm{P}<0.05$ ). (D) Kaplan-Meier method followed by log-rank test was used to analyze the association of FoxP4 expression and the survival rate of HCC patients. HCC, hepatocellular carcinoma.

USA) according to manufacturer's instructions. The firefly luciferase activity was normalized to Renilla luciferase activity. Each independent experiment was replicated at least three times.

Statistical analysis. Each independent experiment was repeated at least three times. Statistical analysis was performed using SPSS software, version 17.0 (SPSS, Inc., Chicago, IL, USA), and all data were presented as the mean \pm SD. A student's t-test was used to determine the statistical significance of the differences between two groups, and one-way analysis of variance followed by Tukey's test was used to analyze the statistical significance of the differences between multiple groups. The Kaplan-Meier method followed by log-rank test was used to plot the survival curves based on FoxP4 relative expression and overall survival. The relationship of FoxP4 expression and pathological characteristics of patients was analyzed by $\chi^{2}$ test. The correlations between the expression of EMT indicator proteins and FoxP4 were analyzed using Spearman's correlation analysis. $\mathrm{P}<0.05$ was considered to indicate a statistically significant difference.

\section{Results}

The expression of FoxP4 is elevated in HCC tissues and cell lines. To investigate the functions of FoxP4 in HCC, we first determined the expression of FoxP4 in HCC tissues and their adjacent normal tissues using RT-qPCR assay, revealing that the expression of FoxP4 was elevated in tumor tissues, compared with adjacent normal tissues (Fig. 1A). In addition, the expression of FoxP4 in HCC cell line HCCLM3 was detected using RT-qPCR and western blotting assays, respectively. The human HL-7702 normal hepatocellular cell line was used as a control. The present results revealed that both mRNA and protein levels of FoxP4 were higher in the HCC cell line than that in normal hepatocytes (Fig. 1B and C). Subsequently, the correlation between the expression of FoxP4 and the clinical information of HCC patients was determined. The mean value of FoxP4 mRNA content in tumor cells was used as the standard. Thus, higher values than the standard value were defined as high expression, and lower values than the standard value were defined as low expression. As revealed in Table I, high expression of FoxP4 was closely associated 
Table I. Clinicopathological variables in $110 \mathrm{HCC}$ patients.

FoxP4 protein expression

\begin{tabular}{|c|c|c|c|c|}
\hline Variables & No. $(n=110)$ & Low $(\mathrm{n}=36)$ & $\operatorname{High}(\mathrm{n}=74)$ & P-value \\
\hline \multicolumn{5}{|l|}{ Sex } \\
\hline Male & 64 & 18 & 46 & 0.225 \\
\hline Female & 46 & 18 & 28 & \\
\hline \multicolumn{5}{|l|}{ Age } \\
\hline$\geq 40$ & 78 & 25 & 53 & 0.814 \\
\hline$<40$ & 32 & 11 & 21 & \\
\hline \multicolumn{5}{|l|}{ Tumor size } \\
\hline Large $(\geq 2 \mathrm{~cm})$ & 62 & 14 & 48 & 0.010 \\
\hline Small $(<2 \mathrm{~cm})$ & 48 & 22 & 26 & \\
\hline \multicolumn{5}{|c|}{ Pathological grade } \\
\hline I-II & 57 & 24 & 33 & 0.030 \\
\hline III-IV & 53 & 12 & 41 & \\
\hline \multicolumn{5}{|c|}{ Lymph node metastasis } \\
\hline Yes & 58 & 14 & 44 & 0.043 \\
\hline No & 52 & 22 & 30 & \\
\hline
\end{tabular}

HCC, hepatocellular carcinoma.

A

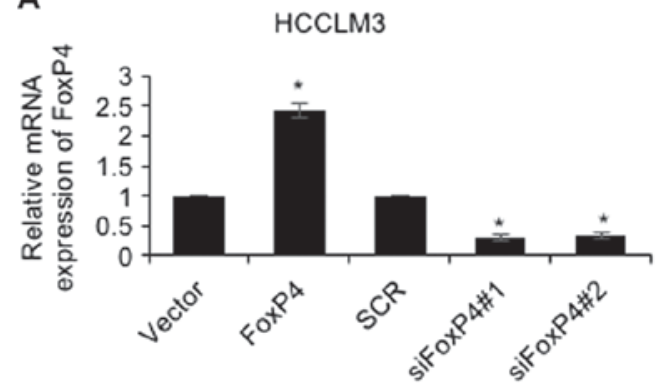

C

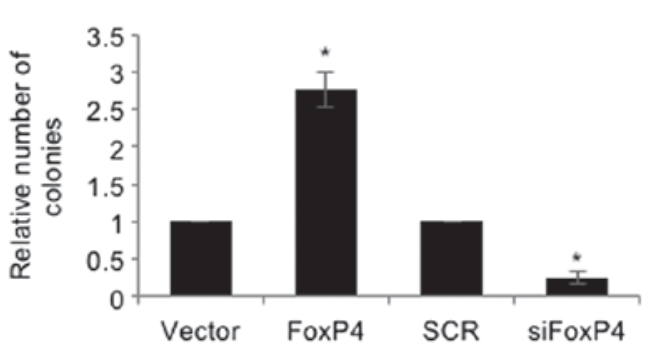

B

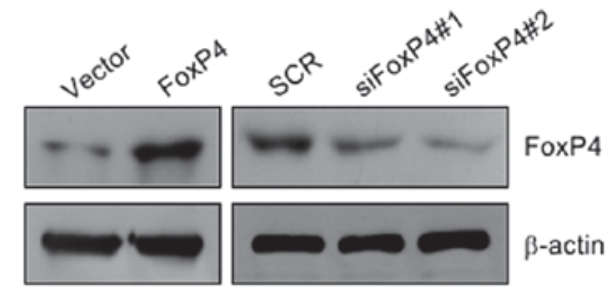

D

HCCLM3

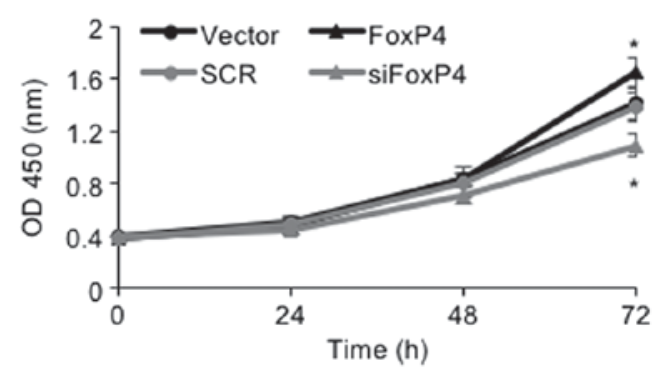

Figure 2. Elevated expression of FoxP4 in HCC cells promotes their proliferation. (A and B) FoxP4 was overexpressed or knocked down in HCCLM3 cells, and after transfection for $48 \mathrm{~h}$, the expression of FoxP4 was determined using RT-qPCR and western blot analyses. (vs. Vector or SCR, "P<0.05). (C) Colony formation assay was used to assess the effect of FoxP4 on HCC cellular proliferation. (vs. Vector or SCR, "P<0.05). (D) CCK-8 assay was used to assess the effect of FoxP4 on HCC cellular proliferation. (vs. Vector or SCR, " $\mathrm{P}<0.05$ ). HCC, hepatocellular carcinoma.

with tumor size, TNM stage and metastasis (Table I), indicating that FoxP4 may play a key role in HCC development. Additionally, analysis of survival data using the Kaplan-Meier method revealed that patients with high expression of FoxP4 had a poor prognosis (Fig. 1D).
Elevated expression of FoxP4 in HCC cells promotes their proliferation. As revealed in Table I, high expression of FoxP4 was closely associated with larger tumor size, thus, we assumed that FoxP4 may play a role in cellular proliferation. To investigate the roles of FoxP4 in HCC, FoxP4 was overexpressed 
A

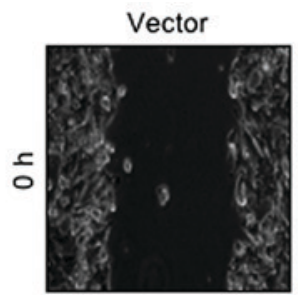

今

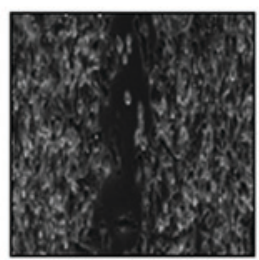

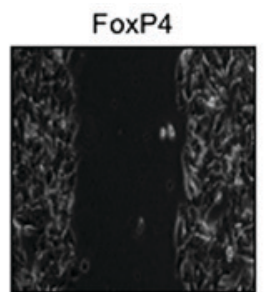

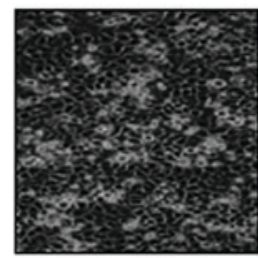

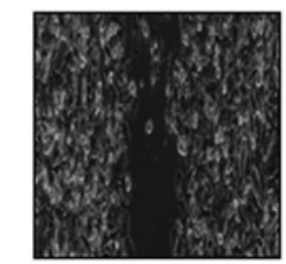

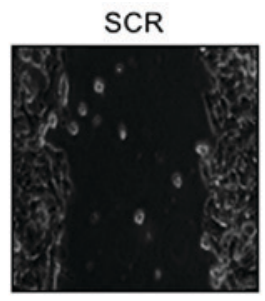

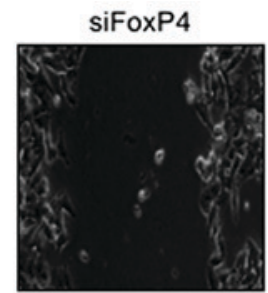

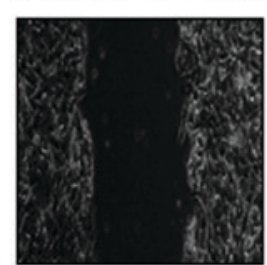

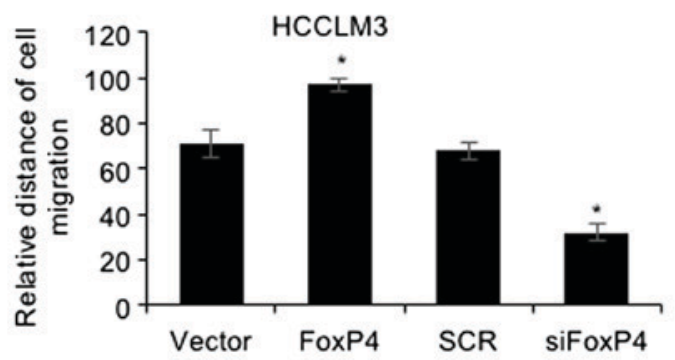

B
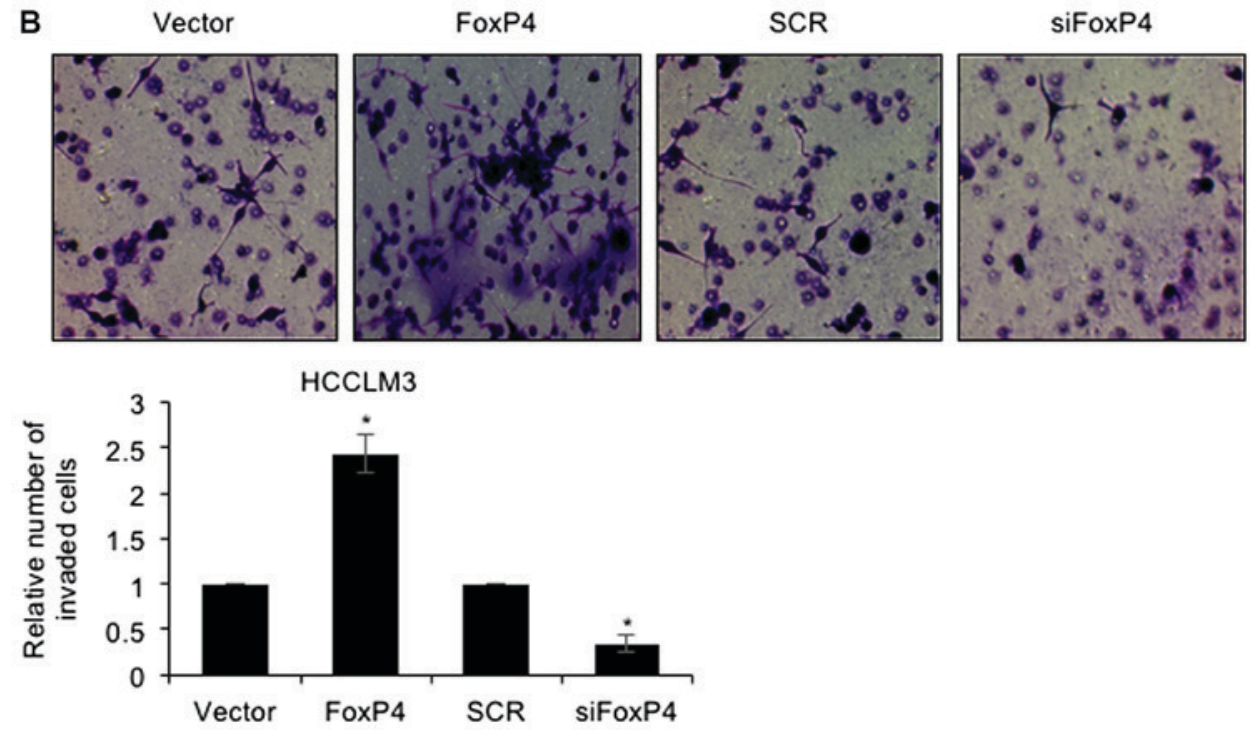

Figure 3. Upregulation of FoxP4 facilitates the migration and invasion of HCC cells. (A) HCCLM3 cells were transfected with pcDNA3.1-FoxP4 plasmid and FoxP4 siRNA. A wound healing assay was utilized to assess the migratory capabilities of HCC cells. (B) A Transwell invasion assay was also performed to assess the invasion capabilities of HCC cells. (vs. Vector or SCR, "P<0.05; magnification, x200). HCC, hepatocellular carcinoma.

or knocked down in HCCLM3 cells using pcDNA3.1-FoxP4 plasmid or siRNA-FoxP4, respectively. RT-qPCR and western blotting assays were utilized to determine the success of the transfection (Fig. 2A and B). Subsequently, colony formation as well as CCK- 8 assays were performed to determine the effect of FoxP4 on cellular proliferation. The results of the colony formation assay revealed that upregulation of the expression of FoxP4 resulted in an elevated number of colonies in HCCLM3 cells compared to the vector group, whereas knockdown of FoxP4 notably reduced the number of colonies compared to the SCR group (Fig. 2C). Additionally, CCK-8 analysis also demonstrated that ectopic expression of FoxP4 improved the cellular proliferation rate and knockdown of FoxP4 impaired the cellular proliferation rate in HCCLM3 cells (Fig. 2D). Collectively, the results indicated that elevated expression of FoxP4 in HCC cells affected cell proliferation.

Upregulation of FoxP4 promotes the migration and invasion of HCC cells. To further decipher the detailed mechanism of FoxP4 in HCC, a wound healing assay and Tranwell invasion assay were performed. The results of the wound healing assay revealed that ectopic expression of FoxP4 potentiated the healing of scratch wounds in HCCLM3 cells, whereas FoxP4 inhibition resulted in the slower healing of scratch wounds in HCCLM3 cells (Fig. 3A), indicating that the migratory capabilities of HCC cells were enhanced following FoxP4 
A

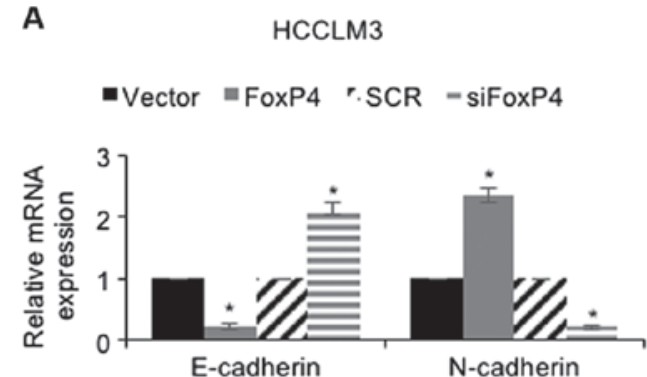

C

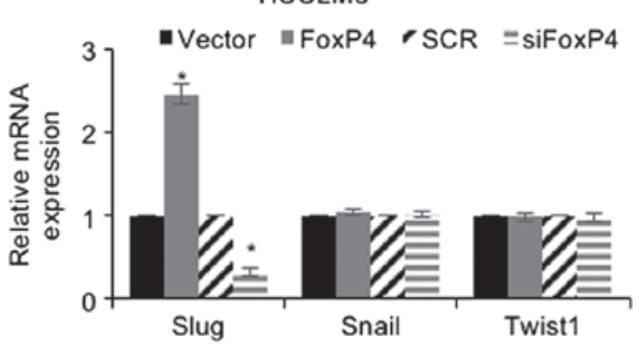

B

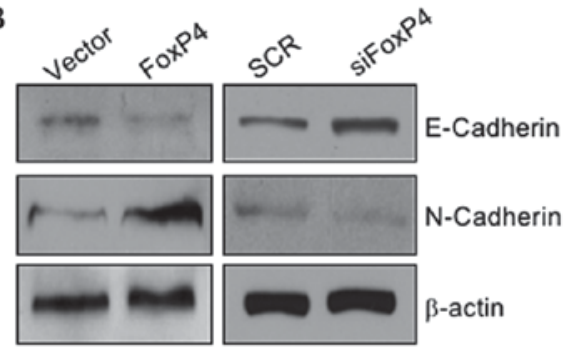

D

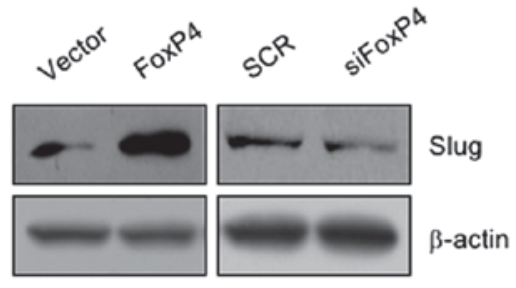

Figure 4. FoxP4 promotes EMT in HCC cells through regulation of Slug. (A and B) HCCLM3 cells were transfected with pcDNA3.1-FoxP4 plasmid and FoxP4 siRNA, and the expression of E-cadherin and N-cadherin were determined using RT-qPCR and western blotting assays. (vs. Vector or SCR, ${ }^{2} \mathrm{P}<0.05$ ). (C and D) HCCLM3 cells were transfected with pcDNA3.1-FoxP4 plasmid and FoxP4 siRNA, and the expression of EMT-associated transcription factors were determined using RT-qPCR and western blotting assays. (vs. Vector or SCR, ${ }^{*} \mathrm{P}<0.05$ ). HCC, hepatocellular carcinoma.

A

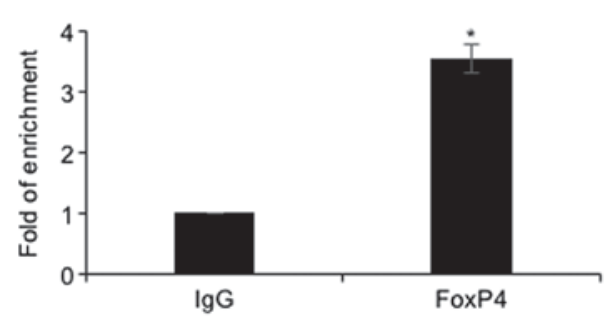

B

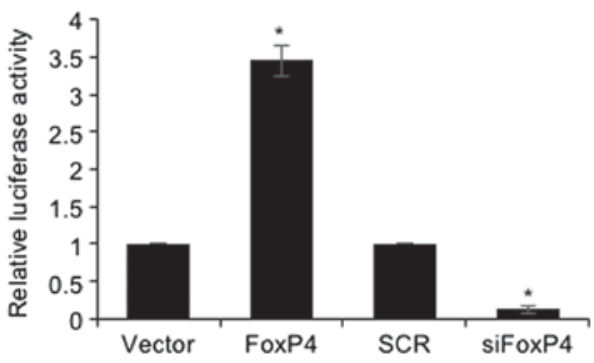

Figure 5. Slug is transcriptionally regulated by FoxP4 in HCC cells. (A) ChIP and qChIP assays were performed using anti-FoxP4 antibody in HCCLM3 cells. (vs. IgG, "P<0.05). (B) 293T cells were transfected with pcDNA3.1-FoxP4 plasmid and FoxP4 siRNA, and a dual luciferase reporter assay was performed. (vs. Vector or SCR, ${ }^{*} \mathrm{P}<0.05$ ). HCC, hepatocellular carcinoma.

overexpression, whereas they were impaired following FoxP4 silencing. For the Transwell invasion assay, the present results revealed that following FoxP4 overexpression, the number of invaded cells was significantly increased compared with the vector cells (Fig. 3B). Conversely, invasive capabilities of siFoxP4-transfected cells were significantly impaired compared with SCR-transfected cells (Fig. 3B). These observations indicated that the upregulation of FoxP4 promoted the migration and invasion of HCC cells.

FoxP4 promotes EMT in HCC cells through regulation of Slug. Multiple studies have revealed that EMT can increase the incidence of cancer metastasis (21-23). Therefore, we ascertained whether FoxP4 promoted the migration and invasion of HCC cells through regulation of EMT. As revealed in Fig. 4A and $\mathrm{B}$, the results of the RT-qPCR assay revealed that following ectopic expression of FoxP4, the mRNA and protein levels of E-cadherin were significantly downregulated and the mRNA and protein levels of $\mathrm{N}$-cadherin were significantly upregulated compared with the vector group (Fig. 4A and B). Conversely, following inhibition of FoxP4, the mRNA and protein levels of
E-cadherin were significantly upregulated and the mRNA and protein levels of $\mathrm{N}$-cadherin were significantly downregulated compared with the SCR group (Fig. 4A and B). The aforementioned results indicated that FoxP4 promoted EMT in HCC cells. Subsequently, whether FoxP4 promoted EMT in HCC cells through regulation of the EMT-associated transcription factors, including Snail, Slug and Twist1, was determined. The present results demonstrated that ectopic expression of FoxP4 resulted in an elevated expression of Slug, whereas inhibition of FoxP4 significantly decreased the expression of Slug; however, neither overexpression nor knockdown of FoxP4 had an effect on Snail and Twistl expression (Fig. 4C and D). These observations indicated that FoxP4 promoted EMT in HCC cells through regulation of Slug.

Slug is transcriptionally regulated by FoxP4 in HCC cells. Since FoxP4 is a transcription factor, it was hypothesized that FoxP4 may transcriptionally regulate Slug. To ascertain our hypothesis, ChIP and qChIP assays were performed, revealing that FoxP4 could directly bind to the promoter region of Slug (Fig. 5A). Subsequently, the promoter region of Slug was cloned 
Table II. Spearman's correlation analysis between the expression of FoxP4 and EMT indicator proteins in HCC.

\section{FoxP4}

expression

\begin{tabular}{lcccc} 
Variables & No. $(\mathrm{n}=110)$ & Low & High & P-value \\
\hline E-cadherin expression & & & & 0.03 \\
$\quad$ High & 57 & 24 & 33 & \\
Low & 53 & 12 & 41 & \\
N-cadherin expression & & & & 0.003 \\
$\quad$ High & 56 & 11 & 45 & \\
Low & 54 & 25 & 29 & \\
Slug expression & & & & 0.042 \\
$\quad$ High & 55 & 13 & 42 & \\
Low & 55 & 23 & 32 & \\
\hline
\end{tabular}

EMT, epithelial-mesenchymal transition; HCC, hepatocellular carcinoma.

into the pGL3-basic plasmid (pGL3-Slug). 293T cells were co-transfected with pGL3-Slug and vector or FoxP4 plasmid, SCR or siFoxP4. The results of the dual luciferase reporter assay demonstrated that ectopic expression of FoxP4 resulted in an elevated relative luciferase activity compared with the vector group, and that in the siFOXP4-transfected cells, the relative luciferase activity was significantly decreased compared with the SCR group (Fig. 5B). Notably, E-cadherin, N-cadherin as well as Slug expression were observed to be correlated with FoxP4 expression in HCC tissues (Table II). Collectively, our results indicated that Slug was transcriptionally regulated by FoxP4 in HCC cells.

\section{Discussion}

In malignant tumors, $>90 \%$ of patients with cancer succumbed to tumor metastasis in 2015 (24). Hepatocellular carcinoma (HCC) is a fast-growing type of cancer that is characterized by highly invasive and metastatic capabilities $(25,26)$. Unfortunately, almost $60-80 \%$ of patients with HCC are diagnosed at an advanced stage, thereby losing the opportunity for surgical treatment (27).

In the present study, FoxP4 was identified to be upregulated in HCC tissues and cells compared with adjacent normal tissues and normal hepatocytes, respectively. Notably, FoxP4 expression was closely associated with tumor size, TNM stage and lymph node metastasis in patients with HCC. Epithelial-mesenchymal transition (EMT) has been revealed to promote cellular invasion and metastasis of cancer (28-30), and the results of our present study revealed that upregulation of FoxP4 significantly promoted the migration and invasion of HCC cells by regulation of EMT. In epithelial cells, E-cadherin is an important protein that regulates cell-cell adhesion (31). The expression of E-cadherin is markedly decreased during EMT, which results in loss of cell-cell adhesion as well as gain of cell motility. Previous studies have indicated that the reduction in
E-cadherin expression is mainly regulated by the transcriptional repressors of E-cadherin gene (CDH1) $(18,32)$. A major transcriptional repressor of CDH1 is the zinc finger factor, Slug (17). Notably, ectopic expression of FoxP4 resulted in an increased expression of Slug. These results indicated that Slug may be involved in FoxP4-mediated EMT.

However, there are a number of limitations present in the study. First, an immunohistochemistry assay is the optimal method to verify the expression of FoxP4 in tissue samples. Second, the roles of FoxP4 in vivo require further investigation. Lastly, the ChIP-seq assay is necessary to extensively investigate FoxP4-target genes in HCC. Further examination is required of the underlying mechanism of FoxP4 in HCC.

In conclusion, our present study revealed that FoxP4 promoted EMT in HCC cells through transcriptional regulation of Slug expression. Moreover, upregulation of FoxP4 also promoted cellular proliferation, thus indicating that FOXP4 may have potential as a novel therapeutic target for the treatment of patients with HCC.

\section{Acknowledgements}

Not applicable.

\section{Funding}

No funding was received.

\section{Availability of data and materials}

The datasets used during the present study are available from the corresponding author upon reasonable request.

\section{Authors' contributions}

GZ and GYZ conceived and designed the present study. GZ and GYZ constructed expression plasmids, prepared proteins and performed experiments. GZ analyzed the data. GZ and GYZ wrote the manuscript. All authors have read and approved the final version of the manuscript.

\section{Ethics approval and consent to participate}

The study was approved by the Ethics Committee of Rizhao Hospital of Traditional Chinese Medicine and each patient was well informed and signed informed consent forms.

\section{Patient consent for publication}

Not applicable.

\section{Competing interests}

The authors declare that they have no competing interests.

\section{References}

1. Waly Raphael S, Yangde Z and Yuxiang C: Hepatocellular carcinoma: Focus on different aspects of management. ISRN Oncol 2012: 421673, 2012. 
2. Forner A, Llovet JM and Bruix J: Hepatocellular carcinoma. Lancet 379: 1245-1255, 2012.

3. Bosch FX, Ribes J, Diaz M and Cleries R: Primary liver cancer: Worldwide incidence and trends. Gastroenterology 127: S5-S16, 2004.

4. Siegel R, Naishadham D and Jemal A: Cancer statistics, 2012. CA Cancer J Clin 62: 10-29, 2012.

5. Faivre S, Bouattour $\mathbf{M}$ and Raymond E: Novel molecular therapies in hepatocellular carcinoma. Liver Int 31 (Suppl): S151-S160, 2011.

6. Pan FC and Wright C: Pancreas organogenesis: From bud to plexus to gland. Dev Dyn 240: 530-565, 2011.

7. Wang X, Sun J, Cui M, Zhao F, Ge C, Chen T, Yao M and Li J: Downregulation of FOXP1 inhibits cell prolife ration in hepatocellular carcinoma by inducing G1/S phase cell cycle arrest. Int J Mol Sci 17: E1501, 2016.

8. Datta J, Kutay H, Nasser MW, Nuovo GJ, Wang B, Majumder S, Liu CG, Volinia S, Croce CM, Schmittgen TD, et al: Methylation mediated silencing of MicroRNA-1 gene and its role in hepatocellular carcinogenesis. Cancer Res 68: 5049-5058, 2008

9. Yan X, Zhou H, Zhang T, Xu P, Zhang S, Huang W, Yang L, $\mathrm{Gu} X$, Ni R and Zhang T: Downregulation of FOXP2 promoter human hepatocellular carcinoma cell invasion. Tumour Biol 36 : 9611-9619, 2015

10. Yu Z, Lin X, Tian M and Chang W: microRNA196b promotes cell migration and invasion by targeting FOXP2 in hepatocellular carcinoma. Oncol Rep 39: 731-738, 2018.

11. Zhang Y, Zhang S, Wang X, Liu J, Yang L, He S, Chen L and Huang J: Prognostic significance of FOXP1 as an oncogene in hepatocellular carcinoma. J Clini Pathol 65: 528-533, 2012.

12. Shi JY, Ma LJ, Zhang JW, Duan M, Ding ZB, Yang LX, Cao Y, Zhou J, Fan J, Zhang X, et al: FOXP3 Is a HCC suppressor gene and Acts through regulating the TGF-beta/Smad2/3 signaling pathway. BMC Cancer 17: 648, 2017.

13. Katoh M and Katoh M: Human FOX gene family (Review) IntJ Oncol 25: 1495-1500, 2004.

14. Myatt SS and Lam EW: The emerging roles of forkhead box (Fox) proteins in cancer. Nat Rev Cancer 7: 847-859, 2007.

15. Lam EW, Brosens JJ, Gomes AR and Koo CY: Forkhead box proteins: Tuning forks for transcriptional harmony. Nat Rev Cancer 13: 482-495, 2013.

16. Barasch J: Genes and proteins involved in mesenchymal to epithelial transition. Curr Opin Nephrol Hypertens 10: 429-436, 2001.

17. Nieto MA: The snail superfamily of zinc-finger transcription factors. Nat Rev Mol Cell Biol 3: 155-166, 2002.

18. Lamouille S, Xu J and Derynck R: Molecular mechanisms of epithelial-mesenchymal transition. Nat Rev Mol Cell Biol 15: 178-196, 2014
19. Lu SQ, Qiu Y, Dai WJ and Zhang XY: FOXR2 promotes the proliferation, invasion, and epithelial-mesenchymal transition in human colorectal cancer cells. Oncol Res 25: 681-689, 2017.

20. Livak KJ and Schmittgen TD: Analysis of relative gene expression data using real-time quantitative PCR and the 2(-Delta Delta C(T)) method. Methods 25: 402-408, 2001.

21. Li J, Xia L, Zhou Z, Zuo Z, Xu C, Song H and Cai J: MiR-186-5p upregulation inhibits proliferation, metastasis and epithelial-to-mesenchymal transition of colorectal cancer cell by targeting ZEB1. Arch Biochem Biophys 640: 53-60, 2018.

22. Sun C, Tao Y, Gao Y, Xia Y, Liu Y, Wang G and Gu Y: F-box protein 11 promotes the growth and metastasis of gastric cancer via PI3K/AKT pathway-mediated EMT. Biomed Pharmacother 98: 416-423, 2018.

23. Liu L, Wu B, Cai H, Li D, Ma Y, Zhu X, Lv Z, Fan Y and Zhang X: Tiam1 promotes thyroid carcinoma metastasis by modulating EMT via Wnt/beta-catenin signaling. Exp Cell Res 362: 532-540, 2018.

24. Guan X: Cancer metastases: Challenges and opportunities. Acta Pharm Sin B 5: 402-418, 2015.

25. Bruix J, Gores GJ and Mazzaferro V: Hepatocellular carcinoma: Clinical frontiers and perspectives. Gut 63: 844-855, 2014.

26. Nordenstedt H, White DL and El-Serag HB: The changing pattern of epidemiology in hepatocellular carcinoma. Dig Liver Dis 42 (Suppl): S206-S214, 2010.

27. Yang JD, Harmsen WS, Slettedahl SW, Chaiteerakij R, Enders FT, Therneau TM, Orsini L, Kim WR and Roberts LR: Factors that affect risk for hepatocellular carcinoma and effects of surveillance. Clin Gastroenterol Hepatol 9: 617-623, 2011.

28. Meng FD, Wei JC, Qu K, Wang ZX, Wu QF, Tai MH, Liu HC, Zhang RY and Liu C: FoxM1 overexpression promotes epithelial-mesenchymal transition and metastasis of hepatocellular carcinoma. World J Gastroenterol 21: 196-213, 2015.

29. Yang J and Weinberg RA: Epithelial-mesenchymal transition: At the crossroads of development and tumor metastasis. Dev Cell 14: 818-829, 2008.

30. Sanchez-Tillo E, Lazaro A, Torrent R, Cuatrecasas M, Vaquero EC, Castells A, Engel P and Postigo A: ZEB1 represses E-cadherin and induces an EMT by recruiting the SWI/SNF chromatin-remodeling protein BRG1. Oncogene 29: 3490-3500, 2010.

31. Tam WL and Weinberg RA: The epigenetics of epithelial-mesenchymal plasticity in cancer. Nature Med 19: 1438-1449, 2013.

32. De Craene B and Berx G: Regulatory networks defining EMT during cancer initiation and progression. Nat Rev Cancer 13: 97-110, 2013 